\title{
The top squark-mediated annihilation scenario and direct detection of dark matter in compressed supersymmetry
}

\author{
Stephen P. Martin \\ Department of Physics, Northern Illinois University, DeKalb IL 60115 and \\ Fermi National Accelerator Laboratory, P.O. Box 500, Batavia IL 60510
}

\begin{abstract}
Top squark-mediated annihilation of bino-like neutralinos to top-antitop pairs can play the dominant role in obtaining a thermal relic dark matter abundance in agreement with observations. In a previous paper, it was argued that this can occur naturally in models of compressed supersymmetry, which feature a running gluino mass parameter that is substantially smaller than the wino mass parameter at the scale of apparent gauge coupling unification. Here I study in some more detail the parameter space in which this is viable, and compare to other scenarios for obtaining the observed dark matter density. I then study the possibility of detecting the dark matter directly in future experiments. The prospects are consistently very promising for a wide variety of model parameters within this scenario.
\end{abstract}

\section{Contents}

I. Introduction

II. Assumptions and parameters

III. Compressed supersymmetry

IV. Direct detection of dark matter neutralinos

V. Outlook

References 


\section{INTRODUCTION}

Supersymmetry provides a candidate for the particle dark matter required by cosmological and astrophysical observations [1], provided that the lightest supersymmetric particle (LSP) is a neutralino and $R$-parity is conserved. Supersymmetry also contains a solution for the hierarchy problem associated with the small ratio of the electroweak breaking scale to the Planck scale and other high scales. The simplest version, the Minimal Supersymmetric Standard Model (MSSM) 2], contains a large undetermined parameter space, due mainly to our present ignorance of the mechanism behind supersymmetry breaking. In recent years, the parameter space of the theory has become increasingly constrained by measurements of and limits on flavor violation, searches for Higgs scalar bosons, the density of dark matter as inferred from cosmology, and ongoing direct searches for superpartners at colliders.

The problem of flavor violation in the MSSM can be solved by assuming that the soft supersymmetry-breaking terms are governed by an organizing principle that respects flavor. In the most general version, this means that at some special input renormalization scale $Q_{0}$, the $3 \times 3$ squared mass matrices for squarks and sleptons with the same electroweak quantum numbers are each (approximately) proportional to the identity matrix:

$$
\mathbf{m}_{\tilde{\mathbf{Q}}}^{\mathbf{2}}=m_{\tilde{Q}, 0}^{2} \mathbf{I}, \quad \mathbf{m}_{\tilde{\mathbf{u}}}^{2}=m_{\tilde{u}, 0}^{2} \mathbf{I}, \quad \mathbf{m}_{\tilde{\mathbf{d}}}^{2}=m_{\tilde{d}, 0}^{2} \mathbf{I}, \quad \mathbf{m}_{\tilde{\mathbf{L}}}^{2}=m_{\tilde{L}, 0}^{2} \mathbf{I}, \quad \mathbf{m}_{\tilde{\mathbf{e}}}^{2}=m_{\tilde{e}, 0}^{2} \mathbf{I} .
$$

Also, in the idealized limit the sfermion-sfermion-Higgs $3 \times 3$ matrix couplings in the Lagrangian are proportional to the corresponding Yukawa couplings:

$$
\mathbf{a}_{\mathbf{u}}=A_{u, 0} \mathbf{y}_{\mathbf{u}}, \quad \mathbf{a}_{\mathbf{d}}=A_{d, 0} \mathbf{y}_{\mathbf{d}}, \quad \mathbf{a}_{\mathbf{e}}=A_{e, 0} \mathbf{y}_{\mathbf{e}}
$$

These assumptions reduce the number of MSSM parameters to only 14 beyond those already found in the Standard Model. The other parameters can be taken to be three independent gaugino masses $M_{1}, M_{2}, M_{3}$, the ratio of Higgs vacuum expectation values $\tan \beta$, and the supersymmetric Higgs mass parameter $\mu$, taken to be real here.

Most of the well-studied scenarios for the MSSM, including minimal supergravity (mSUGRA), gauge-mediated supersymmetry breaking, and anomaly-mediated supersymmetry breaking, are special cases of this flavor-preserving model framework. For example, as popularly applied, mSUGRA makes the further assumptions:

$$
\begin{aligned}
m_{1 / 2} & \equiv M_{1}=M_{2}=M_{3} \\
m_{0}^{2} & \equiv m_{\tilde{Q}, 0}^{2}=m_{\tilde{u}, 0}^{2}=m_{\tilde{d}, 0}^{2}=m_{\tilde{L}, 0}^{2}=m_{\tilde{e}, 0}^{2} \\
A_{0} & \equiv A_{u, 0}=A_{d, 0}=A_{e, 0} .
\end{aligned}
$$

at a scale $Q_{0}$ usually taken to be the scale of apparent gauge coupling unification $M_{\mathrm{GUT}} \approx 2 \times 10^{16}$ $\mathrm{GeV}$. However, it is clear that the parameterization (1.3)-(1.5) may be too simplistic to adequately approximate the real world, even qualitatively.

One of the strongest constraints on the MSSM parameter space comes from the requirement of electroweak symmetry breaking, given the observed value of the top quark mass and the fact that a 
Higgs scalar boson was not discovered at LEP. This requires a balance between the supersymmetrypreserving and supersymmetry-violating contributions to the Higgs scalar squared mass. The apparent tuning required by this balance has become known as the supersymmetric little hierarchy problem.

Another issue is that for many otherwise viable MSSM parameters, the predicted thermal relic abundance of the predicted bino-like LSP is too large, and the universe would have become overclosed (matter dominated too early). Conversely, if the LSP is mostly wino-like or higgsinolike, the predicted dark matter density is far too small to agree with the results of WMAP and other experiments [3]-[5]. The exceptional cases in the mSUGRA version of the MSSM usually fall into four main categories.

First, there is a "bulk region" of parameter space, in which the LSP is mostly bino-like and pair annihilates efficiently due to the $t$-channel and $u$-channel exchange of light sleptons. In much of the mSUGRA parameter space, this possibility has been ruled out or is being squeezed by the searches for the Higgs boson or other superpartners. Second, there is a Higgs resonance region [6], usually found at large $\tan \beta$, in which neutralinos pair annihilate through the $s$-channel exchange of the pseudo-scalar Higgs boson $A^{0}$. Third, the LSP might have a significant higgsino component, allowing efficient neutralino pair annihilation and sometimes co-annihilation with the heavier charginos and neutralinos, to and through weak bosons [7]. This occurs prominently in the "focus point" region of parameter space [8], for large $m_{0}$ in mSUGRA. Fourth, there is a sfermion co-annihilation region [9], where a sfermion (most often a tau slepton [10] in mSUGRA, but possibly a top squark [11]-[16]) happens to be slightly heavier than the LSP. Significant numbers of this sfermion will then coexist with the LSP around the time of freeze-out, so co-annihilations of the sfermion with itself and the LSP will efficiently dilute the superpartners and so the eventual dark matter density.

Recently there have been many studies of dark matter properties that go beyond the mSUGRA assumptions, allowing non-universal scalar masses [17]-[23] or gaugino masses [24]-[42] at the input scale. For example, one can adjust the wino content of the LSP to be big enough to allow for efficient co-annihilations of the LSP with the heavier charginos and neutralinos. It is a common theme of these works that the parameter space in which the thermal relic density of dark matter comes out in agreement with experiment can be significantly enlarged by considering the more general boundary conditions.

In ref. [41], I proposed another remedy with distinctive features, that the LSP is predominantly bino-like and the crucial suppression of the thermal relic density of dark matter is brought about by the process

$$
\tilde{N}_{1} \tilde{N}_{1} \rightarrow t \bar{t}
$$

mostly mediated by $\tilde{t}_{1}$ exchange in the $t$ and $u$ channels. In contrast to the other quark and lepton final states, this channel does not suffer from $p$-wave suppression, because the top quark mass is large. The naturalness of this scenario is based on the supposition that the running gluino mass parameter $M_{3}$ is significantly smaller than the bino and wino mass parameters $M_{1}$ and $M_{2}$ at the scale of apparent gauge unification $M_{\mathrm{GUT}} \approx 2 \times 10^{16} \mathrm{GeV}$. This causes the spectrum of physical superpartner masses to be "compressed" compared to the usual mSUGRA, gauge-mediated, or anomaly-mediated scenarios. If $\left|M_{3}\right|<\left|M_{2}\right|$ at $M_{\mathrm{GUT}}$, then the amount of fine-tuning required of 
other parameters to obtain electroweak symmetry breaking is substantially reduced, as was noted long ago in [43]. This can occur, for example, if the gaugino masses at the apparent GUT scale are governed by supersymmetry-breaking $F$-terms in a combination of a singlet and an adjoint representation of $S U(5)$. It had previously been suggested in models with small $M_{3}$ that the dark matter density can be explained by an enhanced higgsino content of $\tilde{N}_{1}$, providing for enhanced annihilations via $\tilde{N}_{1} \tilde{N}_{1} \rightarrow W^{+} W^{-}$and $Z Z[28,35]$ or by $s$-channel annihilation mediated by the pseudoscalar Higgs $A^{0}$ near resonance [28, 31, 33], by co-annihilations with the heavier higgsino-like charginos and neutralinos [31, 35], or by $s$-channel annihilations to $t \bar{t}$ through the $Z$ boson [28, 33].

In compressed supersymmetry, it can be natural for the lighter top squark, $\tilde{t}_{1}$, to be not much heaver than the LSP and even to be the next-to-lightest supersymmetric particle (NLSP), because renormalization group contributions to $m_{\tilde{t}_{1}}$ from the gluino mass are smaller. This is in distinction to mSUGRA and similar models, in which $\left|A_{0}\right|$ has to be much larger and more finely adjusted in order to arrange for $\tilde{t}_{1}$ to be not much heavier than the LSP [12, 13, 14, 16]. Then $\tilde{N}_{1} \tilde{t}_{1}$ and $\tilde{t}_{1} \tilde{t}_{1}$ co-annihilations are usually also very important in mSUGRA where this can occur, unlike the compressed supersymmetry case discussed here.

Although there is no such thing as an objective measure of fine-tuning, I argued in ref. [41] that for $\left|M_{3}\right| \sim 0.3\left|M_{2}\right|$ at $M_{\mathrm{GUT}}$, this scenario can be considered natural compared to other possibilities discussed in the literature, since a relatively large range of scalar masses gives a prediction for $\Omega_{\mathrm{DM}} h^{2}$ in the range allowed by WMAP and other experiments [3]-[5].

In this paper, I will explore some of the features of the compressed supersymmetry scenario in more detail. Section III provides a more detailed look at the allowed parameter space, concentrating on the case of combined $S U(5)$ singlet and adjoint representation $F$-term supersymmetry breaking. In particular, I will explore how compressed symmetry breaking connects to other mechanisms for obtaining the observed $\Omega_{\mathrm{DM}} h^{2}$, and the dependence on scalar trilinear couplings and $\tan \beta$. In section IV. I study the prospects in compressed supersymmetry for future direct detection of dark matter, which turn out to be quite promising. Section $[\nabla$ contains some concluding remarks.

\section{ASSUMPTIONS AND PARAMETERS}

The parameter space of the MSSM is highly sensitive to the top-quark mass $m_{t}$, especially because of the way it enters into the prediction for the lightest Higgs mass $m_{h}$. The present combined Tevatron result [44] is $m_{t}=170.9 \pm 1.1$ (statistical) \pm 1.5 (systematic). For a fixed lower bound on $m_{h}$, lower $m_{t}$ will exclude more parameter space. In this paper I will be (perhaps) mildly permissive by fixing $m_{t}$ to be at the 1-sigma upper bound, combining the statistical and systematic errors in quadrature:

$$
m_{t}=172.7 \mathrm{GeV} \text {. }
$$

In all of the models discussed below, the lightest Higgs boson has very similar production and decay rates as that of a Standard Model Higgs boson, for which the LEP bound [45] is $114.4 \mathrm{GeV}$. However, I will again be mildly permissive, enforcing only a bound

$$
m_{h}>113.0 \mathrm{GeV}
$$


which takes into account that there remain significant theoretical uncertainties in the prediction for $m_{h}$ for any given set of model parameters.

In the following, the constraint from WMAP and other experiments [3]-[5] on the thermal relic abundance of dark matter is taken to be:

$$
0.09<\Omega_{\mathrm{DM}} h^{2}<0.13
$$

The value of $\Omega_{\mathrm{DM}} h^{2}$ for a given model is obtained by using the program micrOMEGAs 2.0.1 [46] (checked for approximate agreement with DarkSUSY [47]) interfaced to the supersymmetry model parameters program SOFTSUSY 2.0.11 [48] (checked for approximate agreement with SuSpect [49] and ISAJET [50]).

It should also be noted that the MSSM contributions to $\mathrm{BR}(B \rightarrow s \gamma)$ reduce it from the Standard Model prediction 51] of $(3.29 \pm 0.33) \times 10^{-4}$, and can be significant in the models discussed below, leading to an apparent discrepancy with the averaged measured value $(3.55 \pm$ $0.24 \pm 0.10 \pm 0.03) \times 10^{-4}$ for $E_{\gamma}>1.6 \mathrm{GeV}$ [52]. (This has been discussed in more detail in [42].) However, a small amount of flavor violation in the scalar trilinear or squark masses can easily accommodate the measurements, without altering the other predictions of the model in any substantial way. Therefore, this will not be applied as a constraint.

As noted in the Introduction and ref. [41], the essential features of compressed supersymmetry can be realized in a simple one-parameter extension of the well-known mSUGRA model framework. If one assumes that the $F$-term VEVs that break supersymmetry transform as a singlet and an adjoint (24 dimensional) representation of $S U(5)$, then the gaugino masses can be parameterized by [53]-[56]:

$$
\begin{aligned}
& M_{1}=m_{1 / 2}\left(1+C_{24}\right) \\
& M_{2}=m_{1 / 2}\left(1+3 C_{24}\right) \\
& M_{3}=m_{1 / 2}\left(1-2 C_{24}\right) .
\end{aligned}
$$

applied in this paper at $Q=M_{\mathrm{GUT}}$. The special case $C_{24}=0$ yields the mSUGRA (or the minimal gauge-mediated) prediction for gaugino masses. For simplicity, I will also assume a common scalar squared mass $m_{0}^{2}$ and a common scalar trilinear coupling parameter $A_{0}$ at $M_{\mathrm{GUT}}$. The parameter $\mu$ is assumed to be real and positive, in the convention of [2], for a phase choice in which $M_{3}$ is also positive. In the models studied here, that tends to give better agreement with the experimental result for the anomalous magnetic moment of the muon than the Standard Model, although by an amount that is not large compared to the present deviation of up to $3 \sigma$ [5, 57]. Since I am not prepared to claim that the Standard Model is ruled out by the anomalous magnetic moment of the muon, no constraint is applied. However, negative $\mu$ would give a worse agreement than in the Standard Model.

The viability of models considered below requires significant top-squark mixing, and a non-zero $A_{0}$ parameter at $M_{\mathrm{GUT}}$. In the following, I will assume that $A_{0}$ is negative, in the conventions of 2]. The motivation for this is that $A_{0}$ represents the value at $M_{\mathrm{GUT}}$, while the mSUGRA boundary conditions should more properly be applied closer to $M_{\text {Planck }}$. If there is strong renormalization group running between the Planck scale and the apparent GUT scale, it is most likely due to gauge 


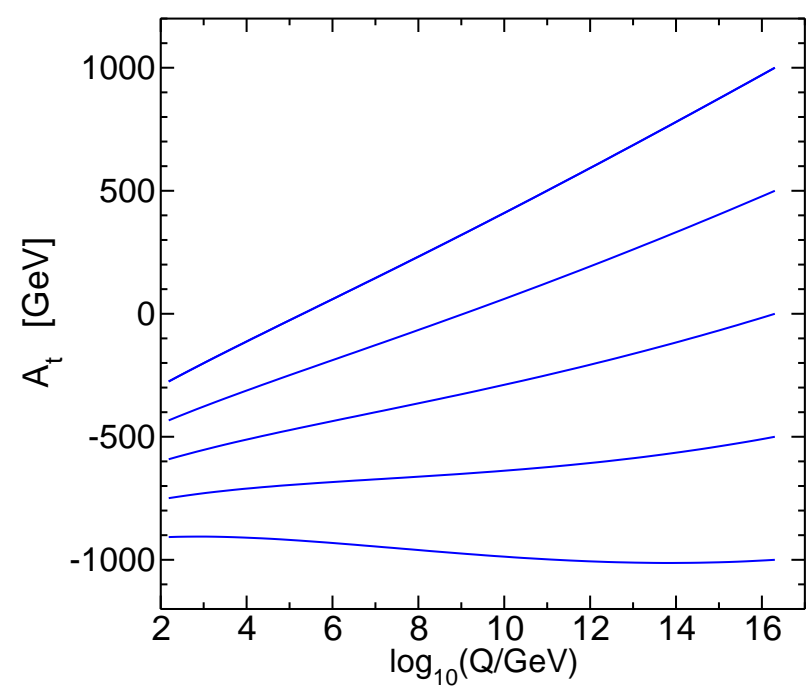

FIG. 1: Running of the parameter $A_{t} \equiv a_{t} / y_{t}$ as a function of the renormalization scale $Q$ in compressed supersymmetry, for various boundary conditions $A_{0} / M_{1}=0, \pm 1, \pm 2$ at $M_{\mathrm{GUT}}$. The parameters are $C_{24}=$ $0.21, M_{1}=500 \mathrm{GeV}$ at $M_{\mathrm{GUT}}, \tan \beta=10$, and $\mu>0$. The renormalization group running causes $A_{t}$ to run with a weak focusing behavior towards negative values at the electroweak scale.

interactions and proportional to the gaugino masses, since this maintains the crucial property of approximate flavor independence. The corresponding renormalization group equations drive the effective parameters $A_{0, u}, A_{0, d}$, and $A_{0, e}$ towards negative values in the infrared at $M_{\mathrm{GUT}}$ where they enter as boundary conditions. It has been argued that large flavor-independent scalar squared masses and trilinear terms can be obtained from an infrared-stable fixed point [58] or an ultravioletstable fixed point [59] behavior above $M_{\mathrm{GUT}}$. From this point of view, it is unclear whether the three parameters $A_{0, u}, A_{0, d}$, and $A_{0, e}$ will actually be unified in the absence of true gauge group unification, but the coupling of top squarks to the Higgs field is of the most direct importance, so I will use a single $A_{0}$ for simplicity. The parameter $A_{t}=a_{t} / y_{t}$ continues to run negative below the unification scale. For the models to be studied below, $A_{t}$ does not approach a true fixed point running, but has a focusing behavior similar to that well-known in mSUGRA [60], as shown in Figure 1. This makes it easy to achieve the required level of top-squark mixing for $A_{0}$ negative and of order $M_{1}$ at the GUT scale, just as expected from [58] or [59] or more generally from any kind of strong running above $M_{\mathrm{GUT}}$. To achieve the right amount of stop mixing with positive $A_{0}$ at $M_{\mathrm{GUT}}$ instead would require a large hierarchy of $A_{0} / M_{1}$ to overcome the tendency of $A_{t}$ to run negative.

In summary, for the sake of simplicity, I will consider models based on the parameters:

$$
m_{1 / 2}, \quad C_{24}, \quad m_{0}, \quad A_{0}, \quad \tan \beta,
$$

imposed at $M_{\mathrm{GUT}}$, with $\mu>0$ in all cases. I expect that similar features will obtain in models with more general boundary conditions, with the gaugino mass ratios being the most essential qualitative feature. 


\section{COMPRESSED SUPERSYMMETRY}

In this section, I present a more detailed look at the compressed MSSM parameter space with $C_{24} \neq 0$ in which the thermal dark matter density constraint is satisfied, augmenting the discussion in ref. [41].

Figure 2 shows the regions allowed by the constraints on $m_{h}$ and $\Omega_{\mathrm{DM}} h^{2}$ in some typical twoparameter model spaces. Here I have chosen $C_{24}=0.21$, which implies approximately $M_{1}: M_{2}$ : $M_{3}:: 2.04: 2.81: 1$ at the apparent unification scale $M_{\mathrm{GUT}}$. The other fixed parameters are $\tan \beta=10, \mu>0$, and three values $A_{0} / M_{1}=-0.6,-1.0$, and -1.5 at $M_{\mathrm{GUT}}$. The parameters $M_{1}$ and $m_{0}$ are free. The bulge regions are where $\tilde{N}_{1} \tilde{N}_{1} \rightarrow t \bar{t}$ makes the dominant contribution to reducing the dark matter density. The left panel shows the allowed values of the lighter top squark mass $\tilde{t}_{1}$, subject to the dark matter constraint of eq. (2.3). The Higgs mass bound eq. (2.2) cuts off the allowed region on the left. This constraint becomes more important for smaller values of $-A_{0} / M_{1}$, cutting away much of the bulge region for the $-A_{0} / M_{1}=0.6$ case. For the thinner regions with $m_{\tilde{N}_{1}}$ above and below the bulge regions, stop co-annihilations (for $-A_{0} / M_{1}=1.0$ and 1.5 ) or stau co-annihilations (for $-A_{0} / M_{1}=0.6$ ) rather than annihilations to top quarks are the crucial factor in limiting the dark matter density.

In the right panel of Figure 2, I show the values of the input parameter $m_{0}$ required for the same models. Here we note the trend that for larger $-A_{0} / M_{1}$, the allowed regions require $m_{0}$ to be larger and in a narrower range. This finer required adjustment may be taken to imply that much larger values of $-A_{0} / M_{1}$ are not as likely. Conversely, for smaller values of $-A_{0} / M_{1}$, the region that would be otherwise allowed is eliminated by the Higgs mass constraint. (Recall that I have already been mildly permissive in this regard.) One can conclude that moderately negative values of $-A_{0} / M_{1}$ at the GUT scale are the most likely realizations of the compressed supersymmetry scenario.

Figure 3 shows similar plots, but this time for the case of $C_{24}=0.24$, which implies the somewhat more severe hierarchy $M_{1}: M_{2}: M_{3}:: 2.38: 3.31: 1$ at the GUT scale. This time, because the ratio of squark to neutralino masses is relatively even smaller, the Higgs mass bound is correspondingly more stringent, and now cuts off a significant part of the $-A_{0} / M_{1}=1.0$ region. The $-A_{0} / M_{1}=0.6$ bulge region does not survive at all, and so has been replaced by $-A_{0} / M_{1}=0.8$. In the right panel, one sees that the values of $m_{0}$ that are required are larger than for the $C_{24}=0.21$ case, but still only of order $500 \mathrm{GeV}$ in the stop-mediated bulge region.

In order to put the scenario illustrated above into a more general context, consider the dark matter allowed regions as $C_{24}$ and $m_{0}$ are allowed to vary. Figure 4 shows the allowed regions as shaded (red) for three fixed values of $M_{1}=-A_{0}=350,500$, and $800 \mathrm{GeV}$, with $\tan \beta=10$ and $\mu>0$. The allowed region in each case consists of a thin strip, topologically forming a ring, with long sections where the qualitative features leading to the correct dark matter density are relatively constant and identifiable.

For $C_{24}=0$ (the mSUGRA case), there are two solutions, one with very large $m_{0}$ of order several $\mathrm{TeV}$, and the other with small $m_{0}$. The large $m_{0}$ solution is the focus point scenario [8], in which $\mu$ is small and the LSP has a significant Higgsino content. As $m_{0}$ is increased beyond this large value, $\mu$ becomes even smaller and there is soon no solution consistent with LEP bounds or electroweak symmetry breaking. The solution for $C_{24}=0$ with small $m_{0}$ is a stau co-annihilation region, in which the mass difference between the LSP and the stau is sufficiently small that they 

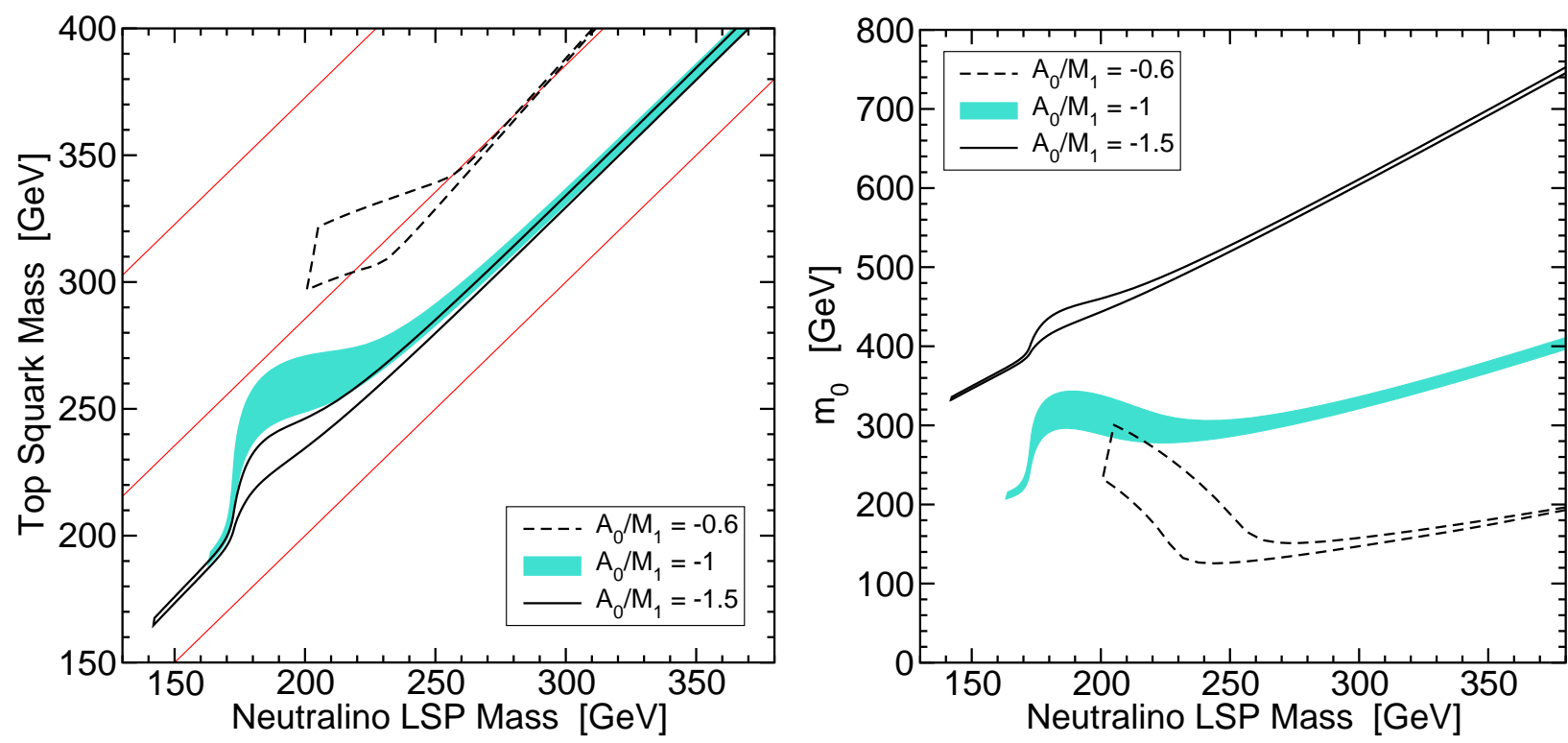

FIG. 2: The regions that satisfy the constraint $0.09<\Omega_{\mathrm{DM}} h^{2}<0.13$ are shown for $C_{24}=0.21$, with $\tan \beta=10, \mu>0$ and varying $M_{1}$ and $m_{0}$. The regions enclosed by dashed lines, shaded, and enclosed by solid lines correspond respectively to $A_{0} / M_{1}=-0.6,-1$, and -1.5 at the unification scale. The left panel shows $m_{\tilde{t}_{1}}$ as a function of the neutralino LSP mass $m_{\tilde{N}_{1}}$. The lowest thin (red) line corresponds to $m_{\tilde{t}_{1}}=m_{\tilde{N}_{1}}$, below which the stop would be the LSP. The middle and upper thin (red) lines bound the regions in which respectively $\tilde{t}_{1} \rightarrow W b \tilde{N}_{1}$ and $\tilde{t}_{1} \rightarrow t \tilde{N}_{1}$ become kinematically allowed. The right panel shows the input parameter $m_{0}$ as a function of the LSP mass for the same regions.
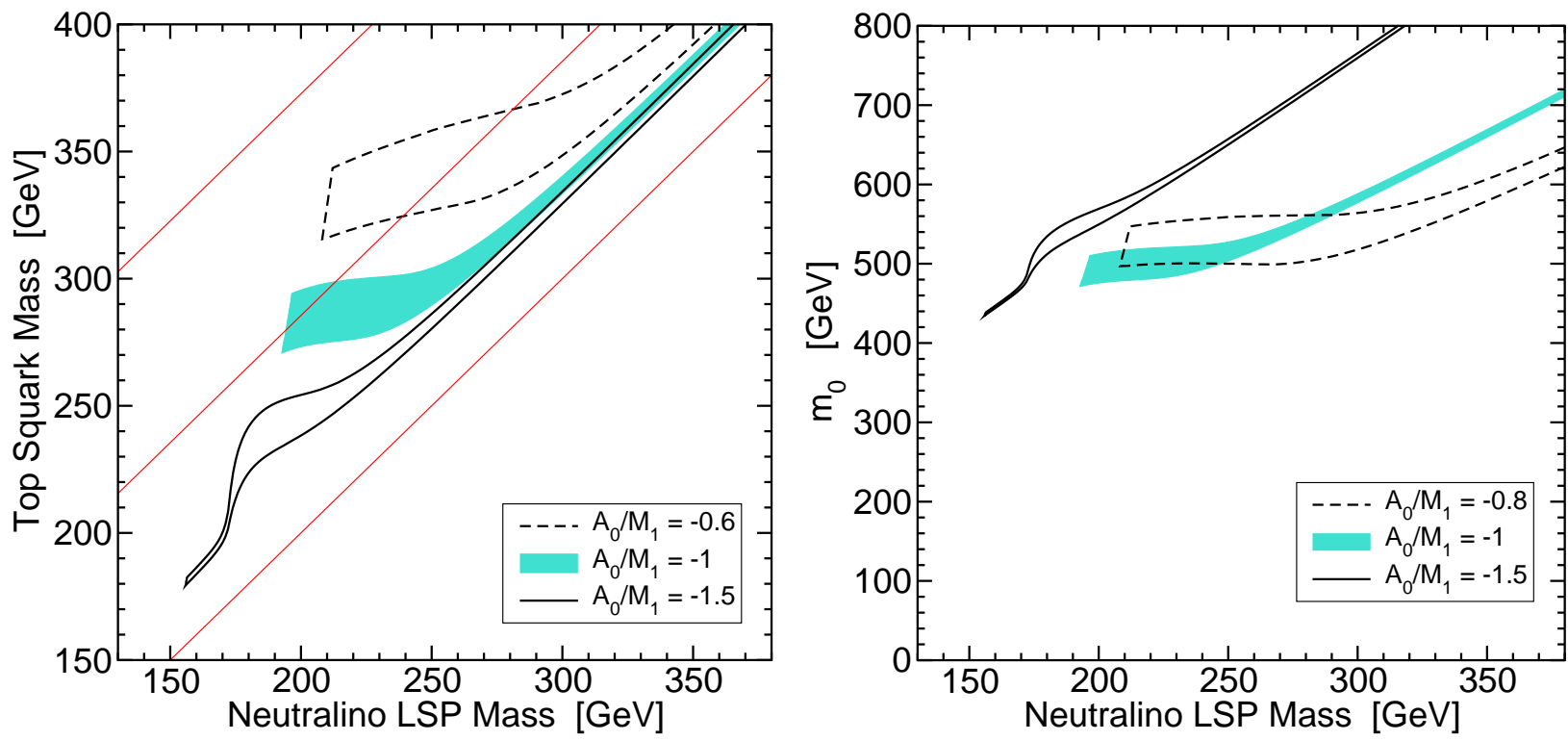

FIG. 3: As in Figure 2, but with $C_{24}=0.24$, and now the regions enclosed by dashed lines, shaded, and enclosed by solid lines correspond respectively to $A_{0} / M_{1}=-0.8,-1$, and -1.5 at the unification scale. 

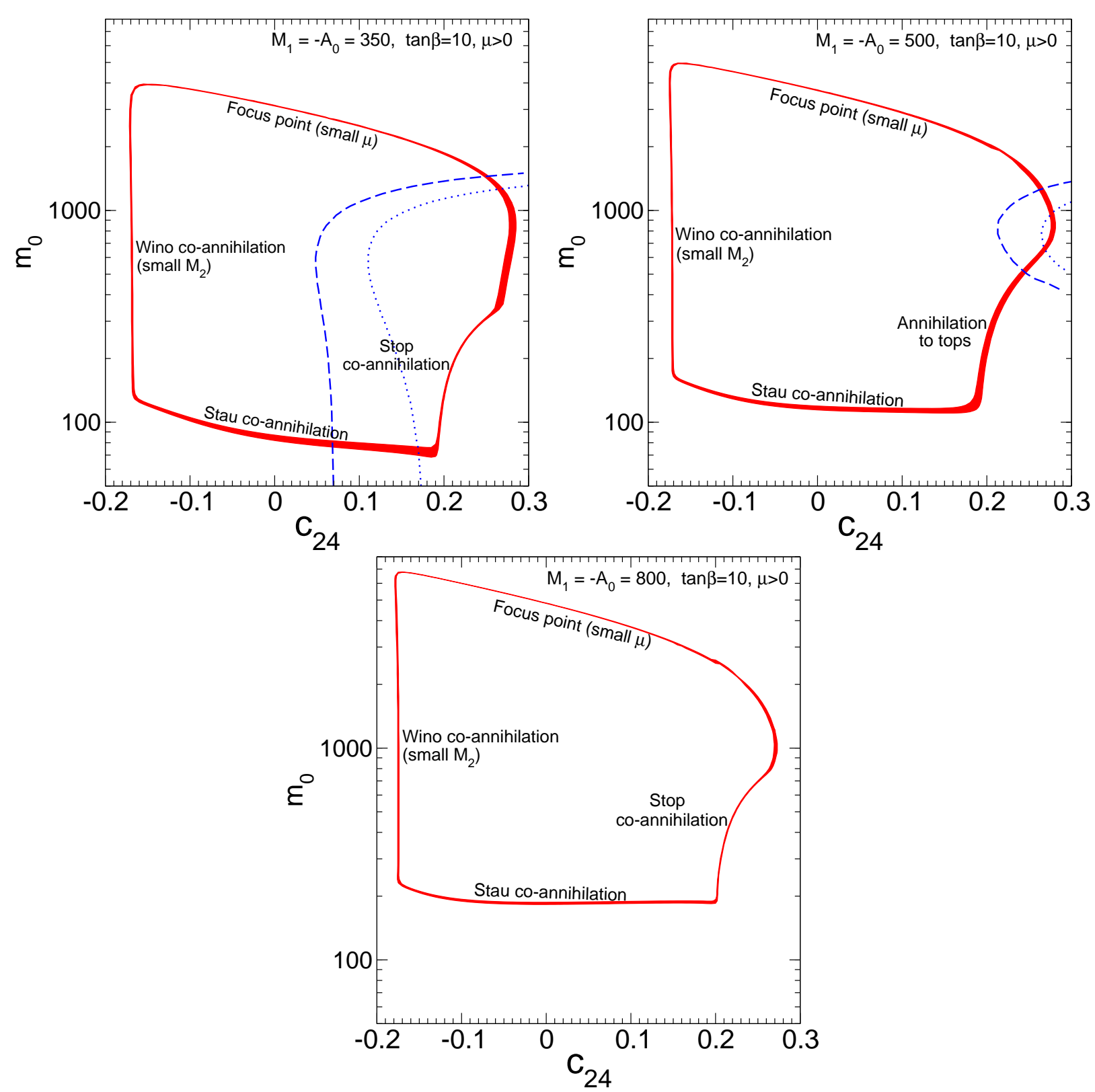

FIG. 4: The shaded (red) regions of parameter space shown are allowed by the thermal relic dark matter density constraint $0.09<\Omega_{\mathrm{DM}} h^{2}<0.13$. The three panels show results for $M_{1}=-A_{0}=350,500$, and $800 \mathrm{GeV}$, with $\tan \beta=10$ and $\mu>0$ in all cases, and with varying common scalar masses $m_{0}$ and gaugino non-universality parameter $C_{24}$. In the $M_{1}=350$ and $M_{1}=500 \mathrm{GeV}$ panels, all points to the right of the dashed (blue) line have a computed value of $m_{h}<113 \mathrm{GeV}$, and all points to the right of the dotted (blue) line have $m_{h}<112 \mathrm{GeV}$. The $M_{1}=500 \mathrm{GeV}$ case has a region in the lower right where $\tilde{N}_{1} \tilde{N}_{1} \rightarrow t \bar{t}$ dominates the determination of $\Omega_{\mathrm{DM}} h^{2}$ without significant assistance from co-annihilations, as suggested in ref. [41].

exist together in thermal equilibrium in the early universe, allowing efficient co-annihilations. Both the focus point and stau co-annihilation regions exist for a significant range of both positive and negative $C_{24}$.

The nearly vertical left side of the allowed region in each case, near $C_{24}=-0.17$ to -0.18 , 
corresponds to $M_{2} / M_{1} \sim 0.6$ near $M_{\mathrm{GUT}}$. This leads to an LSP with a large wino content, which in the allowed region is tuned to give just the right dark matter density. In this region, the co-annihilations of $\tilde{N}_{1} \tilde{C}_{1}$ and $\tilde{C}_{1} \tilde{C}_{1}$ and $\tilde{N}_{1} \tilde{N}_{1}$ and $\tilde{N}_{2} \tilde{C}_{1}$ are all important.

In the lower right part of the $M_{1}=350 \mathrm{GeV}$ panel, there is a thin stop co-annihilation region where the stop is the NLSP. However, the Higgs mass bound, shown as a dashed (blue) line for $m_{h}>113 \mathrm{GeV}$ and a dotted (blue) line for the more optimistic constraint $m_{h}>112 \mathrm{GeV}$, eliminates this region (and the part of the stau co-annihilation region with $C_{24}>0.07$ ) for the smaller $M_{1}$ case.

For the case of intermediate gaugino masses, illustrated in Figure 4 by $M_{1}=500 \mathrm{GeV}$, the stop co-annihilation region is replaced by a fatter region where $\tilde{N}_{1} \tilde{N}_{1} \rightarrow t \bar{t}$ mediated by $\tilde{t}_{1}$ is the dominant mechanism for dark matter suppression. This scenario is the main object of interest here; it is the one that was argued for in [41], and appears as the bulge regions of Figures 2 and 3 , It is important to note that in this region, co-annihilations play only a very small role. Only the upper right corner of this region, where it would join with the focus point region, is eliminated by the Higgs mass constraint.

For sufficiently large $M_{1}$, the LSP becomes too heavy for $\tilde{N}_{1} \tilde{N}_{1} \rightarrow t \bar{t}$ to dominate, and the thin stop co-annihilation region reappears, as illustrated in the lower right part of the $M_{1}=800 \mathrm{GeV}$ panel of 4 . In this case, the $m_{h}$ constraint does not have any impact, but the price to be paid for this is that the tuning required to obtain correct electroweak symmetry breaking and the tuning required to obtain the right amount of dark matter are both worsened.

It should be remarked that in the focus point region with $m_{\tilde{N}_{1}}>m_{t}$, it is often the case that the process $\tilde{N}_{1} \tilde{N}_{1} \rightarrow t \bar{t}$ is also important in regulating the dark matter density, although for a quite different reason; there the top squark is irrelevant, and the $s$-channel $Z$ and Higgs exchange diagrams (due to the higgsino content of the LSP) are the important ones.

In each of the three panels in Figure 4, $\Omega_{\mathrm{DM}} h^{2}$ is predicted to be too large in the unshaded interior region, if the dark matter is due to thermal relics.

For larger $\tan \beta$, the stau co-annihilation region requires larger scalar masses $m_{0}$, since the lighter stau mass is reduced by the effects of the tau Yukawa coupling. This tends to squeeze out the region in which a light stop can play an important role, as $\tilde{N}_{1} \tilde{N}_{1} \rightarrow \tau^{+} \tau^{-}$mediated by staus becomes more important. Furthermore, the annihilation of LSPs through the pseudo-scalar Higgs $A^{0}$ eventually opens up for larger $\tan \beta$, so that the relative importance of $\tilde{N}_{1} \tilde{N}_{1} \rightarrow t \bar{t}$ is further reduced in the dark matter allowed region. Therefore, one expects that for sufficiently large $\tan \beta$, the scenario in which annihilation to top quarks plays the most important role for small or moderate $m_{0}$ will disappear. This is illustrated in Figure 5, where $C_{24}=0.21$ and $A_{0} / M_{1}=-1$ are held fixed, $\mu>0$, and for each value of $M_{1}, m_{0}$ is adjusted to the (lower) value that gives rise to $\Omega_{\mathrm{DM}} h^{2}=0.11$. For various values of $\tan \beta$, the figure shows the relative contribution of the process $\tilde{N}_{1} \tilde{N}_{1} \rightarrow t \bar{t}$ to $1 /\left(\Omega_{\mathrm{DM}} h^{2}\right)$, in per cent, as a function of $m_{\tilde{N}_{1}}$. For $\tan \beta \lesssim 10$, the Higgs mass constraint eliminates viable models unless $m_{\tilde{N}_{1}}$ is sufficiently large. For $\tan \beta \lesssim 5$, the LSP is forced to be so heavy that the annihilation to top quarks is no longer the dominant factor. For $\tan \beta \gtrsim 25$, the annihilation to top quarks is again not the dominant process, and is nearly negligible for $\tan \beta \gtrsim 35$. It is for the intermediate ranges, roughly

$$
\begin{aligned}
& 5 \lesssim \tan \beta \lesssim 25 \\
& m_{t}<m_{\tilde{N}_{1}} \lesssim m_{t}+100 \mathrm{GeV}
\end{aligned}
$$




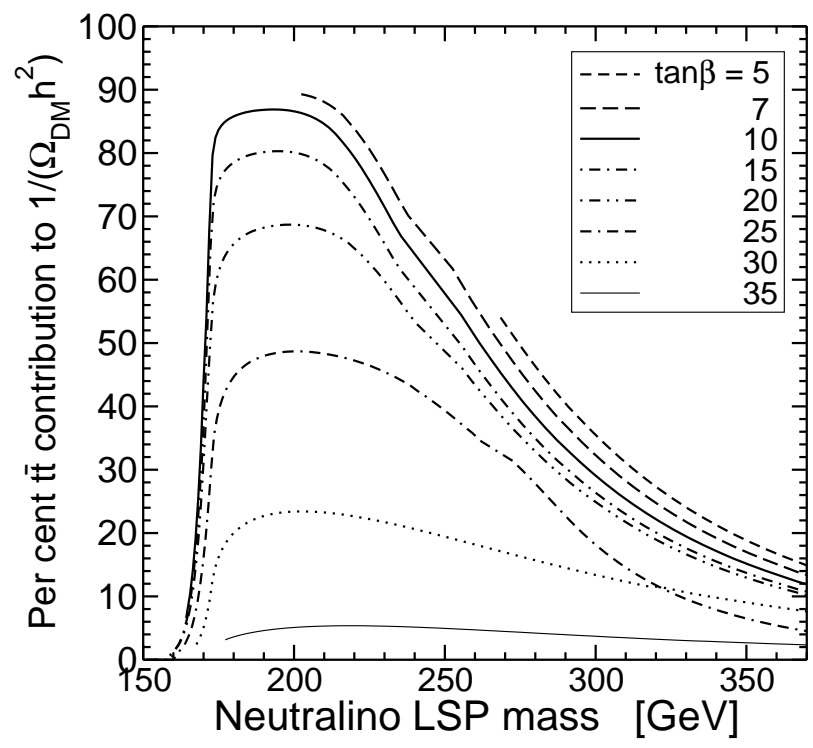

FIG. 5: The relative contribution of the process $\tilde{N}_{1} \tilde{N}_{1} \rightarrow t \bar{t}$ to $1 /\left(\Omega_{\mathrm{DM}} h^{2}\right)$, in per cent, for various values of $\tan \beta$, as a function of $m_{\tilde{N}_{1}}$. In all cases, $C_{24}=0.21, A_{0}=-M_{1}$ at $Q=M_{\mathrm{GUT}}, \mu>0, M_{1}$ is varying, and $m_{0}$ is adjusted to obtain $\Omega_{\mathrm{DM}} h^{2}=0.11$.

$$
m_{\tilde{N}_{1}}+25 \mathrm{GeV} \lesssim m_{\tilde{t}_{1}} \lesssim m_{\tilde{N}_{1}}+150 \mathrm{GeV}
$$

that the stop-mediated annihilation of LSPs can play the most important role.

\section{DIRECT DETECTION OF DARK MATTER NEUTRALINOS}

Dark matter neutralinos can in principle be detected by their weak interaction elastic scattering with ordinary matter nuclei in low-background laboratory detectors. The spin-independent part of the neutralino-nucleon elastic cross-section adds coherently for heavy nuclei, and so gives the best prospects for successful detection. In most cases, the $\tilde{N}_{1} p \rightarrow \tilde{N}_{1} p$ and $\tilde{N}_{1} n \rightarrow \tilde{N}_{1} n$ cross-sections are comparable, and the difference is not large compared to theoretical uncertainties associated with the structure of the proton and neutron. It has therefore become conventional to describe search results and projections in terms of the spin-independent scattering cross-section for the dark matter particles on the proton.

Figure [ shows lines corresponding to present and future projected limits from some dark matter direct detection experiments, assuming a standard local density of $\rho_{\mathrm{LSP}} \approx 0.3 \mathrm{GeV} / \mathrm{cm}^{3}$. The solid lines are the latest results from CDMSII [61] and XENON10 [62]. Projections for two representative next-generation experiments, the XENON100 [63] and SuperCDMS 25kg at SNOLAB [64], appear as dashed lines. The lowest line is the projection for a ton-scale Xenon experiment, XENON1T 63]. The data for these lines came from the compilation and comparison data archive at [65].

Also shown in Figure 6 are the results for the spin-independent LSP-proton cross-section in a variety of compressed supersymmetry models. The spin-independent cross-section

$$
\sigma_{S I}\left(\tilde{N}_{1} p\right)
$$


has been computed using an implementation of the formulas of Drees and Nojiri [66]. It should be noted that the prediction is subject to significant uncertainties that are presently unavoidable, due in part to the lack of accuracy for the values of the quark-antiquark matrix elements for the proton,

$$
f_{T_{q}}^{p}=\left\langle p\left|m_{q} \bar{q} q\right| p\right\rangle / m_{p} \quad(q=u, d, s) .
$$

In the following, I use in particular:

$$
f_{T_{u}}^{p}=0.023, \quad f_{T_{d}}^{p}=0.034, \quad f_{T_{s}}^{p}=0.14
$$

Other values used in the literature can give results for $\sigma_{S I}$ that are higher or lower by a factor of up to 2 or 3 ; see for example [67].

The most important contribution to the spin-independent cross-section in the models examined below always comes from $h^{0}$ (the lighter CP-even neutral Higgs boson) exchange, with $H^{0}$ exchange playing a lesser role and other contributions usually still less. The cross-section is enhanced by the bino-higgsino mixing that is generally a feature of compressed supersymmetry, due to $|\mu|$ not being too large. It has already been noted, for example in [35], that models with relatively small $\left|M_{3}\right|$ tend to have good direct detection prospects for this reason. In the following, I will establish that this holds in some generality in compressed supersymmetry models as parameterized above. Ref. 42] has recently independently found comparable results in a couple of somewhat different compressed supersymmetry model lines. (Ref. [42] also examines indirect detection opportunities, which may be observable but are subject to much larger uncertainties in backgrounds and signals.)

The four panels in Figure [6 have $C_{24}=0.19,0.21,0.24$, and 0.28 . In each panel, $\tan \beta=10$ is fixed, and $\mu>0$. Then $M_{1}$ is varied, and $m_{0}$ is adjusted to the (lower) value that gives $\Omega_{\mathrm{DM}} h^{2}=0.11$. Model points for which $\tilde{N}_{1} \tilde{N}_{1} \rightarrow t \bar{t}$ contributes more than $50 \%$ to $1 /\left(\Omega_{\mathrm{DM}} h^{2}\right)$ are denoted by (blue) X's, and those for which $\tilde{N}_{1} \tilde{N}_{1} \rightarrow t \bar{t}$ contributes less than $50 \%$ are denoted by (red) dots. The Higgs mass constraint is imposed, which cuts off the model points to the left side and above. The resulting smallest allowed values of $-A_{0} / M_{1}$ at $M_{\mathrm{GUT}}$ are $(0.1,0.4,0.7,1.1)$ for $C_{24}=(0.19,0.21,0.24,0.28)$, respectively, and correspond to the highest lines of models. Each lower line of model points corresponds to an increase of $-A_{0} / M_{1}$ by 0.1 , up to $-A_{0} / M_{1}=1.6$. Each lower line of models then has $-A_{0} / M_{1}$ increased by 0.2 , up to a limit of $-A_{0} / M_{1}=(4.0,3.8,3.4,3.4)$ for $C_{24}=(0.19,0.21,0.24,0.28)$. These upper limits on $-A_{0} / M_{1}$ follow from the requirement of a consistent model solution with a stable vacuum and $\Omega_{\mathrm{DM}} h^{2}=0.11$.

The spin-independent elastic cross-section can clearly be seen to be larger for smaller $-A_{0}$. This is because smaller $-A_{0}$ corresponds to smaller $\mu$, and therefore enhanced bino-higgsino mixing in the LSP. Likewise, larger values of $C_{24}$ imply a larger cross-section.

I have checked that repeating the analysis of Figure 6 but with larger values of $\tan \beta$ gives even larger cross-sections, while taking smaller values of $\tan \beta$ gives cross-sections that are about the same (or only slightly smaller). For the models of most interest here, in which $\tilde{N}_{1} \tilde{N}_{1} \rightarrow t \bar{t}$ contributes more than $50 \%$ to $1 /\left(\Omega_{\mathrm{DM}} h^{2}\right)$, the cross-sections can be up to about 4 times larger (but often less) than shown in Figure 6. However, for fixed values of the other parameters, increasing $\tan \beta$ within this class of models never raises the cross-section enough to conflict with the present experimental limit from XENON10. 

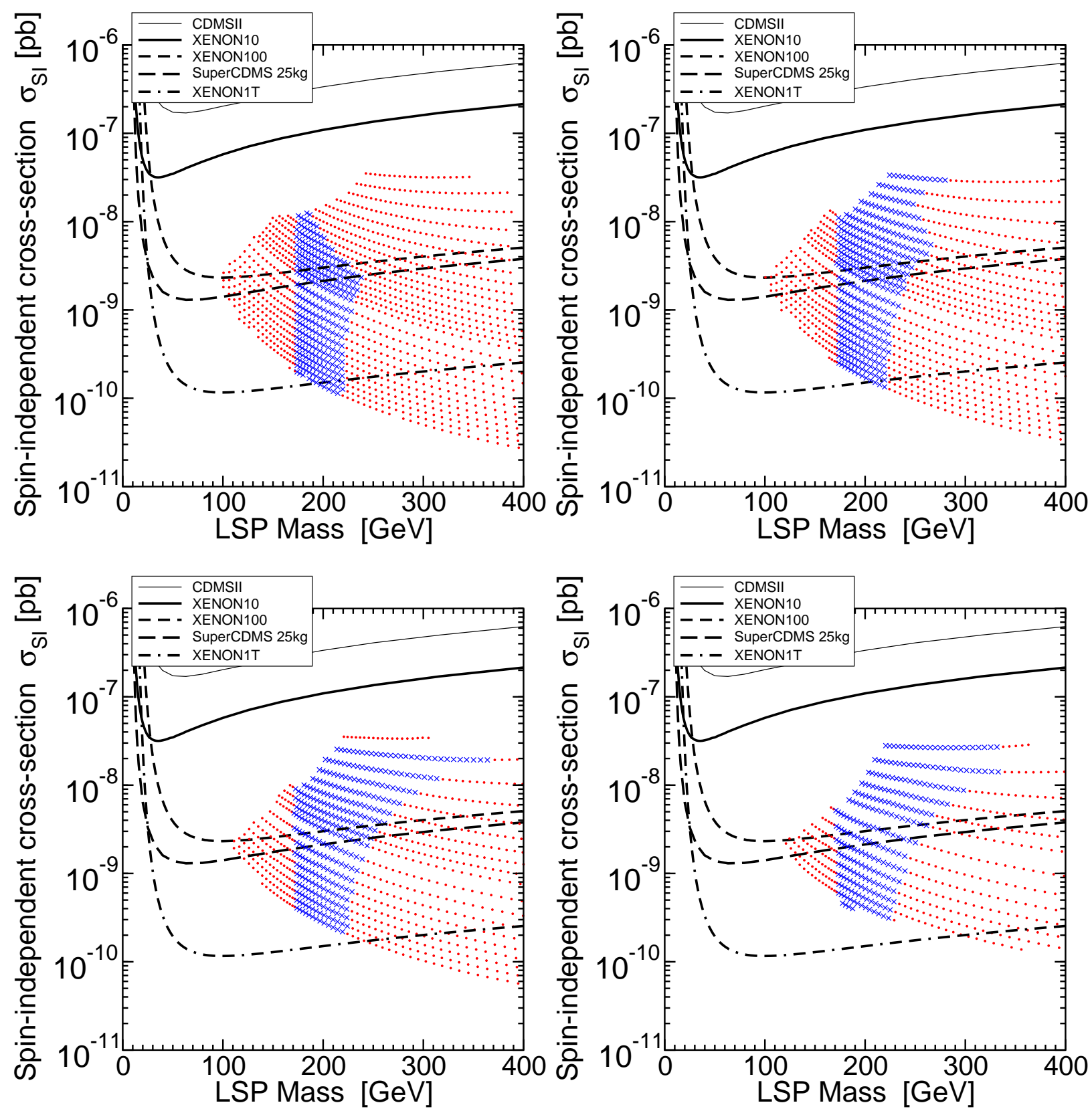

FIG. 6: Spin-independent proton-LSP cross-sections in compressed supersymmetry models, compared to existing limits (solid lines) and some future projected reaches (broken lines) from direct detection experiments. Models in which $\tilde{N}_{1} \tilde{N}_{1} \rightarrow t \bar{t}$ contributes at least 50 per cent to $1 /\left(\Omega_{\mathrm{DM}} h^{2}\right)$ are denoted by (blue) X's, and other models satisfying the WMAP constraints but with $\tilde{N}_{1} \tilde{N}_{1} \rightarrow t \bar{t}$ contributing less than 50 per cent to $1 /\left(\Omega_{\mathrm{DM}} h^{2}\right)$ are denoted by (red) dots. The four panels correspond to $C_{24}=0.19$ (upper left), 0.21 (upper right), 0.24 (lower left), and 0.28 (lower right). For each model, $\tan \beta=10, \mu>0$, and $m_{0}$ is adjusted to the (lower) value such that $\Omega_{\mathrm{DM}} h^{2}=0.11$. The highest line of models in each of the $C_{24}=(0.19,0.21,0.24,0.28)$ panels corresponds to $-A_{0} / M_{1}=(0.1,0.4,0.7,1.1)$ respectively. Each lower line of models has $-A_{0} / M_{1}$ increased by 0.1 until $-A_{0} / M_{1}=1.6$, and then $-A_{0} / M_{1}$ increases by 0.2 for each lower line of models. The lowest line of models for $C_{24}=(0.19,0.21,0.24,0.28)$ has $-A_{0} / M_{1}=(4.0,3.8,3.4,3.4)$, respectively. These upper bounds on $-A_{0} / M_{1}$, which set the lower limits on the cross-section, follow from the requirement of a consistent model solution with a stable vacuum and $\Omega_{\mathrm{DM}} h^{2}=0.11$. The lower bounds on $-A_{0} / M_{1}$ in each panel come from the requirement $m_{h}>113 \mathrm{GeV}$. 
It is apparent that the next generation of experiments has a good chance to detect the dark matter in these models, particularly if $M_{1}$ and $-A_{0} / M_{1}$ are not too large, as suggested by subjective naturalness criteria. Even taking into account the significant uncertainties in the local density of dark matter and the proton matrix elements mentioned above, it appears that the ton-scale XENON1T or similar experiments should be able to definitively test almost all of the considered models where $\tilde{N}_{1} \tilde{N}_{1} \rightarrow t \bar{t}$ makes more than a $50 \%$ contribution to $1 /\left(\Omega_{\mathrm{DM}} h^{2}\right)$, assuming the local dark matter density and velocity distributions are not significantly different from expectations. The exceptions are the models with the largest possible allowed values of $-A_{0} / M_{1}>3.4$ for $C_{24}=0.19$ or 0.21. However, as observed in the previous section, such models can be reasonably considered less likely to be realized in nature, since they require relatively larger and more finely tuned $\mu$. For the seemingly more natural case $-A_{0} / M_{1} \lesssim 1.5$, the XENON100 and SuperCDMS experiments have a chance to probe most of the parameter space, particularly for smaller LSP masses.

These results are in contrast to stau co-annihilation models, which tend to have a much lower spin-independent cross-section. Focus point models usually have a comparable or somewhat larger cross-section to the ones shown in Figure 6.

\section{OUTLOOK}

Compressed supersymmetry, featuring a ratio of the heaviest to lightest superpartner masses that is reduced compared to mSUGRA and other models, can naturally explain the thermal relic abundance of dark matter in the universe while ameliorating the problem of fine-tuning in the electroweak symmetry breaking sector of the MSSM. The dark matter abundance is related both to the existence of a top squark with mass not far above the LSP mass and to a $\mu$ parameter that is not too large while maintaining a predominantly bino-like LSP. This scenario has distinctive discovery signals at the Large Hadron Collider, as noted in [41] and studied in more detail in [42], and should be discovered despite relatively lepton-poor channels due to the near decoupling of sleptons and wino-like charginos from superpartner decay chains. Unfortunately, prospects for study at a future $e^{+} e^{-}$linear collider are likely to be severely limited in this scenario [41] unless the center of mass energy is considerably larger than $\sqrt{s}=500 \mathrm{GeV}$.

In this paper, I have studied the possibility of direct detection of dark matter in the top-squark mediated neutralino annihilation case, with results consistent with those independently obtained recently in [42]. The current limits from CDMS and XENON-10 do not impact on the scenario at all. However, I have found that the prospects in a wide variety of models of this type look quite promising for the next generation of experiments, for models with $-A_{0} / M_{1}$ smaller than roughly 1.5, which seem to be the least fine-tuned. Better, a 1-ton class XENON experiment (or a comparable one) should have the necessary reach to detect neutralino scattering events, even taking into account uncertainties in the cross-section and the local density of dark matter, except in some parts of the model parameter space that would require quite severe fine-tuning. One may therefore anticipate that dark matter direct detection will be quite useful in confirming and clarifying future LHC results if this scenario is correct.

Acknowledgments: This work was supported in part by the National Science Foundation grant 
number PHY-0456635.

[1] G. Jungman, M. Kamionkowski and K. Griest, Phys. Rept. 267, 195 (1996) hep-ph/9506380, G. Bertone, D. Hooper and J. Silk, Phys. Rept. 405, 279 (2005) hep-ph/0404175.

[2] A review that uses the same notations and conventions as here is S.P. Martin, "A supersymmetry primer," hep-ph/9709356, version 4 June 2006.

[3] D.N. Spergel et al. [WMAP Collaboration], "Wilkinson Microwave Anisotropy Probe (WMAP) three year results: Implications for cosmology," astro-ph/0603449.

[4] M. Tegmark et al. [SDSS Collaboration], Phys. Rev. D 69, 103501 (2004) astro-ph/0310723.

[5] W.M. Yao et al. [Particle Data Group], "Review of particle physics," J. Phys. G 33, 1 (2006).

[6] M. Drees and M.M. Nojiri, Phys. Rev. D 47, 376 (1993) hep-ph/9207234.

[7] J. Edsjö and P. Gondolo, Phys. Rev. D 56, 1879 (1997) hep-ph/9704361.

[8] J.L. Feng, K.T. Matchev and F. Wilczek, Phys. Lett. B 482, 388 (2000) hep-ph/0004043, K.L. Chan, U. Chattopadhyay and P. Nath, Phys. Rev. D 58, 096004 (1998) hep-ph/9710473, J.L. Feng, K.T. Matchev and T. Moroi, Phys. Rev. Lett. 84, 2322 (2000) hep-ph/9908309; Phys. Rev. D 61, 075005 (2000) hep-ph/9909334.

[9] K. Griest and D. Seckel, Phys. Rev. D 43, 3191 (1991).

[10] J.R. Ellis, T. Falk and K.A. Olive, Phys. Lett. B 444, 367 (1998) hep-ph/9810360; J.R. Ellis, T. Falk, K.A. Olive and M. Srednicki, Astropart. Phys. 13, 181 (2000) [Erratum-ibid. 15, 413 (2001)] hep-ph/9905481.

[11] M.E. Gomez, G. Lazarides and C. Pallis, Phys. Rev. D 61, 123512 (2000) hep-ph/9907261;

[12] C. Boehm, A. Djouadi and M. Drees, Phys. Rev. D 62, 035012 (2000) hep-ph/9911496;

[13] J.R. Ellis, K.A. Olive and Y. Santoso, Astropart. Phys. 18, 395 (2003) hep-ph/0112113;

[14] J. Edsjo, M. Schelke, P. Ullio and P. Gondolo, JCAP 0304, 001 (2003) hep-ph/0301106, J. Edsjo, M. Schelke and P. Ullio, JCAP 0409, 004 (2004) astro-ph/0405414.

[15] C. Balazs, M. Carena and C.E.M. Wagner, Phys. Rev. D 70, 015007 (2004) hep-ph/0403224.

[16] G. Belanger, F. Boudjema, S. Kraml, A. Pukhov and A. Semenov, Phys. Rev. D 73, 115007 (2006) hep-ph/0604150].

[17] V. Berezinsky et al., Astropart. Phys. 5, 1 (1996) hep-ph/9508249].

[18] P. Nath and R. Arnowitt, Phys. Rev. D 56, 2820 (1997) hep-ph/9701301.

[19] H. Baer, C. Balazs, M. Brhlik, P. Mercadante, X. Tata and Y. Wang, Phys. Rev. D 64, 015002 (2001) hep-ph/0102156.

[20] J.R. Ellis, T. Falk, K.A. Olive and Y. Santoso, Nucl. Phys. B 652, 259 (2003) hep-ph/0210205 J.R. Ellis, A. Ferstl, K.A. Olive and Y. Santoso, Phys. Rev. D 67, 123502 (2003) hep-ph/0302032. J.R. Ellis, K.A. Olive, Y. Santoso and V.C. Spanos, Phys. Lett. B 603, 51 (2004) hep-ph/0408118.

[21] H. Baer, A. Mustafayev, S. Profumo, A. Belyaev and X. Tata, Phys. Rev. D 71, 095008 (2005) hep-ph/0412059.

[22] A. De Roeck et al., "Supersymmetric benchmarks with non-universal scalar masses or gravitino dark matter," hep-ph/0508198.

[23] J.L. Evans, D.E. Morrissey and J.D. Wells, Phys. Rev. D 75, 055017 (2007) hep-ph/0611185.

[24] A. Corsetti and P. Nath, Phys. Rev. D 64, 125010 (2001) [hep-ph/0003186].

[25] A. Birkedal-Hansen and B.D. Nelson, Phys. Rev. D 64, 015008 (2001) hep-ph/0102075], Phys. Rev. D 67, 095006 (2003) hep-ph/0211071.

[26] U. Chattopadhyay, A. Corsetti and P. Nath, Phys. Rev. D 66, 035003 (2002) hep-ph/0201001.

[27] H. Baer, C. Balazs, A. Belyaev, R. Dermisek, A. Mafi and A. Mustafayev, JHEP 0205, 061 (2002) hep-ph/0204108.

[28] V. Bertin, E. Nezri and J. Orloff, JHEP 0302, 046 (2003) hep-ph/0210034.

[29] U. Chattopadhyay and D.P. Roy, Phys. Rev. D 68, 033010 (2003) hep-ph/0304108.

[30] D.G. Cerdeño and C. Muñoz, JHEP 0410, 015 (2004) hep-ph/0405057.

[31] G. Belanger, F. Boudjema, A. Cottrant, A. Pukhov and A. Semenov, Nucl. Phys. B 706, 411 (2005) hep-ph/0407218. 
[32] G. Belanger, F. Boudjema, A. Cottrant, A. Pukhov and A. Semenov, Czech. J. Phys. 55, B205 (2005) hep-ph/0412309].

[33] Y. Mambrini and E. Nezri, Eur. Phys. J. C 50, 949 (2007) hep-ph/0507263.

[34] S.F. King and J.P. Roberts, JHEP 0609, 036 (2006) hep-ph/0603095]; JHEP 0701, 024 (2007) hep-ph/0608135.

[35] H. Baer, A. Mustafayev, E.K. Park, S. Profumo and X. Tata, JHEP 0604, 041 (2006) hep-ph/0603197, H. Baer, A. Mustafayev, S. Profumo and X. Tata, Phys. Rev. D 75, 035004 (2007) hep-ph/0610154].

[36] N. Arkani-Hamed, A. Delgado and G.F. Giudice, Nucl. Phys. B 741, 108 (2006) [hep-ph/0601041].

[37] A. Falkowski, O. Lebedev and Y. Mambrini, JHEP 0511, 034 (2005) hep-ph/0507110.

[38] H. Baer, E.K. Park, X. Tata and T.T. Wang, JHEP 0608, 041 (2006) hep-ph/0604253]; "Collider and Dark Matter Phenomenology of Models with Mirage Unification," hep-ph/0703024.

[39] K.J. Bae, R. Dermisek, H.D. Kim and I.W. Kim, "Mixed bino-wino-higgsino dark matter in gauge messenger models," hep-ph/0702041.

[40] S.F. King, J.P. Roberts and D.P. Roy, "Natural Dark Matter in SUSY GUTs with Non-universal Gaugino Masses," [hep-ph/0705.4219].

[41] S.P. Martin, "Compressed supersymmetry and natural neutralino dark matter from top squarkmediated annihilation to top quarks," Phys. Rev. D 75, 115005 (2007) hep-ph/0703097.

[42] H. Baer, A. Box, E. Park and X. Tata, "Implications of compressed supersymmetry for collider and dark matter searches", [hep-ph/0707.0618].

[43] G.L. Kane and S.F. King, Phys. Lett. B 451, 113 (1999) hep-ph/9810374, M. Bastero-Gil, G.L. Kane and S.F. King, Phys. Lett. B 474, 103 (2000) hep-ph/9910506.

[44] The Tevatron Electroweak Working Group for the CDF and D0 collaborations, "A combination of CDF and D0 results on the mass of the top quark," hep-ex/0703034].

[45] R. Barate et al. [LEP Working Group for Higgs boson searches], Phys. Lett. B 565, 61 (2003) hep-ex/0306033. S. Schael et al. [LEP Working Group for Higgs boson searches], Eur. Phys. J. C 47, 547 (2006) hep-ex/0602042.

[46] G. Belanger, F. Boudjema, A. Pukhov and A. Semenov, "micrOMEGAs2.0: A program to calculate the relic density of dark matter in a generic model," Comput. Phys. Commun. 176, 367 (2007) hep-ph/0607059, Comput. Phys. Commun. 174, 577 (2006) hep-ph/0405253, Comput. Phys. Commun. 149, 103 (2002) hep-ph/0112278.

[47] P. Gondolo, J. Edsjö, P. Ullio, L. Bergstrom, M. Schelke and E.A. Baltz, "DarkSUSY: Computing supersymmetric dark matter properties numerically," JCAP 0407, 008 (2004) astro-ph/0406204;

[48] B.C. Allanach, "SOFTSUSY: A C++ program for calculating supersymmetric spectra," Comput. Phys. Commun. 143, 305 (2002) hep-ph/0104145.

[49] A. Djouadi, J.L. Kneur and G. Moultaka, "SuSpect: A Fortran code for the supersymmetric and Higgs particle spectrum in the MSSM," hep-ph/0211331.

[50] F.E. Paige, S.D. Protopopescu, H. Baer and X. Tata, "ISAJET 7.69: A Monte Carlo event generator for p p, anti-p p, and e+ e- reactions," hep-ph/0312045.

[51] K.G. Chetyrkin, M. Misiak and M. Munz, Phys. Lett. B 400, 206 (1997) [Erratum-ibid. B 425, 414 (1998)] hep-ph/9612313; A.J. Buras, A. Kwiatkowski and N. Pott, Phys. Lett. B 414, 157 (1997) [Erratum-ibid. B 434, 459 (1998)] [hep-ph/9707482]; A.L. Kagan and M. Neubert, Eur. Phys. J. C 7, 5 (1999) hep-ph/9805303.

[52] E. Barberio et al. [Heavy Flavor Averaging Group Collaboration], "Averages of b-hadron properties at the end of 2006," [hep-ex 0704.3575].

[53] J.R. Ellis, K. Enqvist, D.V. Nanopoulos and K. Tamvakis, Phys. Lett. B 155, 381 (1985).

[54] M. Drees, Phys. Lett. B 158, 409 (1985).

[55] G. Anderson, C.H. Chen, J.F. Gunion, J.D. Lykken, T. Moroi and Y. Yamada, "Motivations for and implications of non-universal GUT-scale boundary conditions for soft SUSY-breaking parameters," report for Snowmass 96, hep-ph/9609457.

[56] G. Anderson, H. Baer, C. h. Chen and X. Tata, Phys. Rev. D 61, 095005 (2000) hep-ph/9903370.

[57] G.W. Bennett et al. [Muon G-2 Collaboration], Phys. Rev. D 73, 072003 (2006) [hep-ex/0602035].

[58] I. Jack and D.R.T. Jones, Phys. Lett. B 349, 294 (1995) hep-ph/9501395]. I. Jack, D.R.T. Jones and K.L. Roberts, Nucl. Phys. B 455, 83 (1995) hep-ph/9505242. M. Lanzagorta and G.G. Ross, Phys. Lett. B 364, 163 (1995) hep-ph/9507366. 
[59] S.P. Martin and J.D. Wells, Phys. Rev. D 64, 036010 (2001) hep-ph/0011382.

[60] M. Carena, M. Olechowski, S. Pokorski and C.E.M. Wagner, Nucl. Phys. B 419, 213 (1994) hep-ph/9311222, M. Carena and C.E.M. Wagner, Nucl. Phys. B 452, 45 (1995) hep-ph/9408253, S.A. Abel and B. Allanach, Phys. Lett. B 415, 371 (1997) hep-ph/9707436.

[61] D.S. Akerib et al. [CDMS Collaboration], Phys. Rev. Lett. 96, 011302 (2006) astro-ph/0509259.

[62] J. Angle et al. [XENON Collaboration], "First Results from the XENON10 Dark Matter Experiment at the Gran Sasso National Laboratory," [astro-ph/0706.0039].

[63] E. Aprile et al., "XENON: A 1-tonne liquid xenon experiment for a sensitive dark matter search," astro-ph/0207670; Nucl. Phys. Proc. Suppl. 138, 156 (2005) astro-ph/0407575].

[64] D.S. Akerib et al., "The SuperCDMS proposal for dark matter detection," Nucl. Instrum. Meth. A 559, 411 (2006).

[65] Direct dark matter detection experiment limits and projections may be found at http://dendera.berkeley.edu/plotter/and in the directory plotter/limitdata.

[66] M. Drees and M. Nojiri, "Neutralino - Nucleon Scattering Revisited," Phys. Rev. D 48, 3483 (1993) hep-ph/9307208.

[67] A. Bottino, F. Donato, N. Fornengo and S. Scopel, Astropart. Phys. 13, 215 (2000) hep-ph/9909228. J.R. Ellis, K.A. Olive, Y. Santoso and V.C. Spanos, Phys. Rev. D 71, 095007 (2005) hep-ph/0502001.

[68] J. Gasser, H. Leutwyler and M. E. Sainio, Phys. Lett. B 253, 252 (1991). 